DOI: http://doi.org/10.21698/simi.2018.ab31

\title{
BIOINDICATORS IN AIR QUALITY CONTROL
}

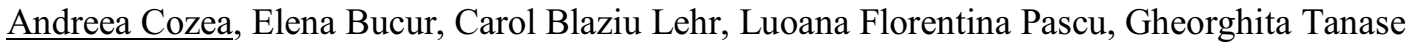

National Research and Development Institute for Industrial Ecology, 71-73 Drumul Podu

Dambovitei, district 6, 060652, Bucharest, andreea.cozea@yahoo.com, Romania

Keywords: air pollution, biomonitoring, foliar necrosis, ozone

\section{Introduction}

Air pollution has become a major environmental problem facing the world today due rapid increase in industrialization and anthropogenic activities. As such, there is need for reliable and sustainable air pollution monitoring and control methods. Biological monitoring of air pollutants can be passive or active. Passive methods observe plants growing naturally within the area of interest. Active methods detect the presence of air pollutants by placing test plants of known response and genotype into the study area. Biomonitoring of air pollution using plants is the method of interest in recent time as it is cost effective, sustainable and environmentally friendly compared to traditional methods.

The use of bioindicators to monitor air pollution and implicitly, ecosystems exposed to pollution phenomena can provide a timely intervention to protect the integrity of the environment and indirectly the health of the human population.

The aim of this study was identification of some specific species of plants and selection of certain plant species with the purpose of use of selected plant species in experimental biomonitoring studies.

\section{Materials and methods}

Some plant species are highly sensitive to particular air pollutants and show specific responses to pollutants effects by showing specific damage symptoms. Plant species were used to detect and monitor air pollutants correlated with foliar necrosis produces by a high ozone level. For this fact, we have proposed to initiate the research on the use of sentinel species. The tests were carried out in this first phase to identify the sensitivity of some plant species of the Solanaceae class: Nicotiana rustica, Nicotiana tabacum, Nicotiana alata and Petunia hibrida.

The tests included three main steps: i) preparation for monitoring, ii) exposure of the test plants in two zones: i) industrial area (I) and green peri-urban area, and iii) exposure effect in the tested areas compared with laboratory controls. Seeds used for sowing were of a physical purity of at least $94 \%$, so a germinative capacity over $70 \%$. The optimal temperature germination temperature of seeds was 28 degrees C. Selected plants were growing in two-liter pots, with standard soil, in laboratory conditions; suitable irrigation was maintained by capillarity. So, eight-week-old cultivars, six plants from each species, were exposed on the exposure racks, for two weeks in two sites, with differing tropospheric ozone levels, from May to June, 2018. Concentration of ozone levels in the tested areas was also measured. In both field areas, the percentages of leaf affected surface by typical ozone-induced necrosis of the fourth, fifth and sixth oldest leaves were estimated by a single operator. Histo-anatomical 


\section{INTERNATIONAL SYMPOSIUM "THE ENVIRONMENT AND THE INDUSTRY", SIMI 2018, BOOK OF ABSTRACTS}

analysis of health and affected by ozone leaves, was used and gives us the opportunity to study the interaction between plants and the environment and to select the suitable sensitive plant type.

\section{Results and conclusions}

Biomonitoring assessment capabilities offer a wide range of information. The information provided is polyvalent, expressing air quality and reproducing the effects of pollution. In our study, visible symptoms such as necrotic areas were observed on the leaves of the exposed plants. The evaluation was done weekly and the percentage of foliar destruction was estimated. From the histo-anatomical analysis from the comparative microscopic images between the Probes and the Controls, we observed that the changes on the stomata characteristics were correlated with the increased of ozone concentration in the environment through previous studies (issued in the Standards) and confirmed in this study. So, changes in the density, distribution and morphology of the stomata on the foliar surface were observed, as well as the occurrence of "necrotic" spots on the foliar level.

Following the tests, we made, we conclude:

In industrial area the number of leaves affected by foliar necrosis was much higher compared to the green peri-urban area. Similarly, the affected foliar surface was higher for plants exposed in industrial area.

A given "response" of plants to the action of the polluted environment was in the following order:

Petunia hibrida (Petunia) $>$ Nicotiana rustica (Wild Tobacco) $>$ Nicotiana tabacum (Tobacco). Nicotiana alata (Queen of the Night) did not respond properly due to the natural fragility of tissues and leave necrosis with atypical format for ozone pollution. In conclusion three bioindicators were selected: Petunia hibrida (Petunia), Nicotiana rustica (Wild Tobacco) and Nicotiana tabacum (Tobacco) to be used in the next step, to continue the study initiated in the first stage by increasing the number and variety of exposure areas, the use of larger plant samples and the monitoring of a larger number of air pollutants. The results obtained will be treated and interpreted statistically to better understand the mechanisms underlying the effects of air pollution on plants.

The plants that are sensitive to a particular pollutant or mixture of pollutants can be used as veritable tools towards mitigating environmental and health problems associated with air pollution. 